\title{
Durable Response of Spinal Chordoma to Combined Inhibition of IGF-1R and EGFR
}

\author{
Tamara Aleksic ${ }^{1}$, Lisa Browning ${ }^{2}$, Martha Woodward ${ }^{3}$, Rachel Phillips ${ }^{4}$, Suzanne Page ${ }^{5}$, \\ Shirley Henderson ${ }^{5}$, Nicholas Athanasou ${ }^{6}$, Olaf Ansorge ${ }^{7}$, Duncan Whitwell ${ }^{6}$, \\ Sarah Pratap ${ }^{3}$, A. Bassim Hassan ${ }^{3}$, Mark R. Middleton ${ }^{3}$ and Valentine M. Macaulay 1,3*

\begin{abstract}
Department of Oncology, Old Road Campus Research Building, Oxford, UK, ${ }^{2}$ Department of Cellular Pathology, NIHR ${ }^{4}$ Department of Radiology, Oxford Cancer and Haematology Centre, Churchill Hospital, Oxford University Hospitals NHS Foundation Trust, Oxford, UK, ${ }^{5}$ BRC Oxford Molecular Diagnostic Centre, John Radcliffe Hospital, Oxford University Hospitals NHS Foundation Trust, Oxford, UK, ${ }^{6}$ Nuffield Department of Orthopaedics, Rheumatology and Musculoskeletal Science, Nuffield Orthopaedic Centre, Oxford, UK, ${ }^{7}$ Nuffield Department of Clinical Neurosciences, John Radcliffe Hospital, Oxford, UK
\end{abstract} \\ Oxford Biomedical Research Centre, John Radcliffe Hospital, Oxford University Hospitals NHS Foundation Trust, Oxford, UK, \\ ${ }^{3}$ Oxford Cancer and Haematology Centre, Churchill Hospital, Oxford University Hospitals NHS Foundation Trust, Oxford, UK,
}

\section{OPEN ACCESS}

Edited by:

Giuseppe Giaccone,

Georgetown University, USA

Reviewed by:

Giuseppe Giaccone,

Georgetown University, USA

Yisong Wang,

National Institute of Health, USA

*Correspondence:

Valentine M. Macaulay valentine.macaulay@oncology.ox.

ac.uk

Specialty section: This article was submitted to Cancer Molecular Targets and Therapeutics, a section of the journal

Frontiers in Oncology

Received: 15 February 2016 Accepted: 07 April 2016 Published: 02 May 2016

Citation: Aleksic T, Browning L, Woodward M, Phillips R, Page S, Henderson S, Athanasou N, Ansorge O, Whitwell D, Pratap S, Hassan AB, Middleton MR and Macaulay VM (2016) Durable Response of Spinal Chordoma to Combined Inhibition of IGF-1R and

EGFR.

Front. Oncol. 6:98. doi: 10.3389/fonc.2016.00098
Chordomas are rare primary malignant bone tumors arising from embryonal notochord remnants of the axial skeleton. Chordomas commonly recur following surgery and radiotherapy, and there is no effective systemic therapy. Previous studies implicated receptor tyrosine kinases, including epidermal growth factor receptor (EGFR) and type 1 insulin-like growth factor receptor (IGF-1R), in chordoma biology. We report an adult female patient who presented in 2003 with spinal chordoma, treated with surgery and radiotherapy. She underwent further surgery for recurrent chordoma in 2008, with subsequent progression in pelvic deposits. In June 2009, she was recruited onto the Phase I OSI-906-103 trial of EGFR inhibitor erlotinib with linsitinib, a novel inhibitor of IGF-1R/insulin receptor (INSR). Treatment with $100 \mathrm{mg}$ QD erlotinib and $50 \mathrm{mg}$ QD linsitinib was well-tolerated, and after 18 months a partial response was achieved by RECIST criteria. From 43 months, a protocol modification allowed intra-patient linsitinib dose escalation to $50 \mathrm{mg}$ BID. The patient remained stable on trial treatment for a total of 5 years, discontinuing treatment in August 2014. She subsequently experienced further disease progression for which she underwent pelvic surgery in April 2015. Analysis of DNA extracted from 2008 (pre-trial) tissue showed that the tumor harbored wild-type EGFR, and a PIK3CA mutation was detected in plasma, but not tumor DNA. The 2015 (post-trial) tumor harbored a mutation of uncertain significance in ATM, with no detectable mutations in other components of a 50 gene panel, including EGFR, PIK3CA, and TP53. By immunohistochemistry, the tumor was positive for brachyury, the molecular hallmark of chordoma, and showed weak-moderate membrane and cytoplasmic EGFR. IGF-1R was detected in the plasma membrane and cytoplasm and was expressed more strongly in recurrent tumor than the primary. We also noted heterogeneous nuclear IGF-1R, which has been linked with sensitivity to IGF-1R inhibition. Similar variation in IGF-1R expression and subcellular localization was noted in 15 further cases of chordoma. In summary, this exceptionally 
durable response suggests that there may be merit in evaluating combined IGF-1R/ INSR and EGFR inhibition in patients with chordomas that recur following failure of local treatment.

Keywords: chordoma, IGF-1R, EGFR, tyrosine kinase inhibitor, nuclear IGF-1R

\section{INTRODUCTION}

Chordomas are rare primary malignant bone tumors arising from primitive notochord remnants, and account for $\sim 17 \%$ of primary malignant bone tumors of the axial skeleton (1). Chordomas express the transcription factor brachyury $(\mathrm{T})$ that directs notochord development (2). Germ-line duplication of the brachyury gene is associated with familial chordoma, copy number gain is reported in sporadic chordomas, and common genetic variants in brachyury have recently been associated with both familial and sporadic chordomas (3-6).

Clinically, there is approximately equal distribution between cranial, spinal, and sacral origin, with risk of local recurrence in $\sim 45 \%$, metastasis in $\sim 30 \%$, and median survival of 6.3 years $(7-9)$. The mainstay of treatment is local therapy in the form of surgery and radiotherapy, ideally with proton beam therapy (9). Invasion of adjacent muscles is common, compromising the likelihood of complete surgical resection, and extent of resection, surgical margins, tumor grade and proton therapy are the most important predictors of recurrence and survival (10-12). Although chordomas are typically indolent, recurrent tumors can pursue a more aggressive course with distant metastases. In a series of 18 sacral chordoma patients, 12 patients experienced recurrence, and of those, 7 died in median 5.4 months (range 4-40 months) after the recurrence (10). Chordomas are resistant to chemotherapy, and there are no licensed options for systemic therapy, although several targeted agents have induced responses in individual cases (13).

\section{DESCRIPTION OF CASE}

The patient is a 65 -year-old non-smoker who presented in mid2003 with a 9-month history of lower back pain. Magnetic resonance imaging (MRI) revealed replacement of the L4 vertebra by a lobulated tumor that invaded the spinal canal, encroaching on the cauda equina. MRI and computed tomography (CT) scans showed no evidence of primary tumor elsewhere. She underwent biopsy under fluoroscopic control followed by surgery on 16th July 2003. The procedure involved radical tumor resection with insertion of an intervertebral cage containing a biphasic calcium phosphate ceramic (Triosite). Histological examination (Figures 1A,B) showed tumor composed predominantly of physaliphorous cells arranged in nests and set in a mucinous matrix. There was considerable nuclear pleomorphism and hyperchromasia and occasional atypical mitoses. Tumor cells were positive for cytokeratins and S100 (not shown), and also for brachyury (Figure 1C), the molecular hallmark of chordoma (2). The tumor infiltrated soft tissue and extended to the surgical margins. Proton therapy being unavailable (14), she underwent a course of adjuvant photon beam radiotherapy to the lumbar spine (L3-L5 inclusive, 50 Gy total dose in 30 fractions).
From April 2007, the patient experienced recurrent pain, weakness, and altered sensation in the lower limbs. Initial MRI scans were equivocal for tumor recurrence but, in August 2007, showed definite evidence of recurrence to the left of the fourth lumbar vertebral body, eroding the superior end-plate and the posterosuperior border of L5 vertebral body. She experienced further clinical and radiological progression, including an acute episode of paraplegia in December 2007, and in February 2008, she underwent major revision surgery. Laparotomy revealed considerable recurrent tumor behind the body of L4 with bleeding into the spinal canal at this level, and tumor infiltrating the psoas muscles, also with hemorrhagic change. She underwent a debulking procedure with anterior vertebrectomy and anterior and posterior fusion, leaving residual tumor in the psoas muscles. Histopathological analysis confirmed recurrent brachyury-positive chordoma in the L4 vertebral body, epidural surface, psoas muscles bilaterally, and left abdominal wall. Postoperatively, there was gradual recovery of power in the lower limbs, although with persistent altered sensation in the legs and loss of knee and ankle reflexes. MRI scans in March 2009 showed further evidence of progression, prompting referral to our Early Phase Clinical Trials Unit in June 2009.

The patient was recruited onto the Phase I OSI-906-103 trial, evaluating linsitinib (OSI-906), a novel inhibitor of the type 1 insulin-like growth factor receptor (IGF-1R) and insulin receptor (INSR), in combination with the epidermal growth factor receptor (EGFR) inhibitor erlotinib (15). Initial investigations showed that hematology, renal and liver function, coagulation screen, fasting glucose, and $\mathrm{HbAlc}$ were all normal, with LDH 232 U/L (normal 100-190 U/L). Baseline CT scan in June 2009 showed extensive bone destruction and surgical fixation involving the lumbosacral spine. MRI, on the same date, showed recurrent tumor with measurable components within the right iliopsoas muscle (mid-right psoas $35 \mathrm{~mm}$, Figure 2A; right side of $\mathrm{L} 3 / 4,29 \mathrm{~mm}$ ) and to the left side of the intervertebral device at L4 $(35 \mathrm{~mm})$. The patient was treated on the S2 (daily linsitinib) schedule (15), receiving $50 \mathrm{mg}$ linsitinib QD from 30th June 2009, with addition of $100 \mathrm{mg}$ erlotinib QD after 1 week. The treatment was well-tolerated aside from dry skin (grade 1), abnormal hair and eyelash growth (grade 1), and bilateral hallux paronychia (grade 2 at worst), attributed to erlotinib, and diarrhea (grade 1), attributed to both agents. There was no evidence of hematologic toxicity, hyperglycemia, or hyperinsulinemia, and no biochemical abnormalities with the exception of creatine kinase, which was measured for the first time in March 2012 and was elevated (333 U/L, normal range 24-195 U/L; grade 1 elevation), with subsequent persistent elevation (350-520, grade 2) probably attributable to psoas muscle involvement.

Sequential MRI scans revealed initial disease stabilization, with gradual improvement to partial response by response 


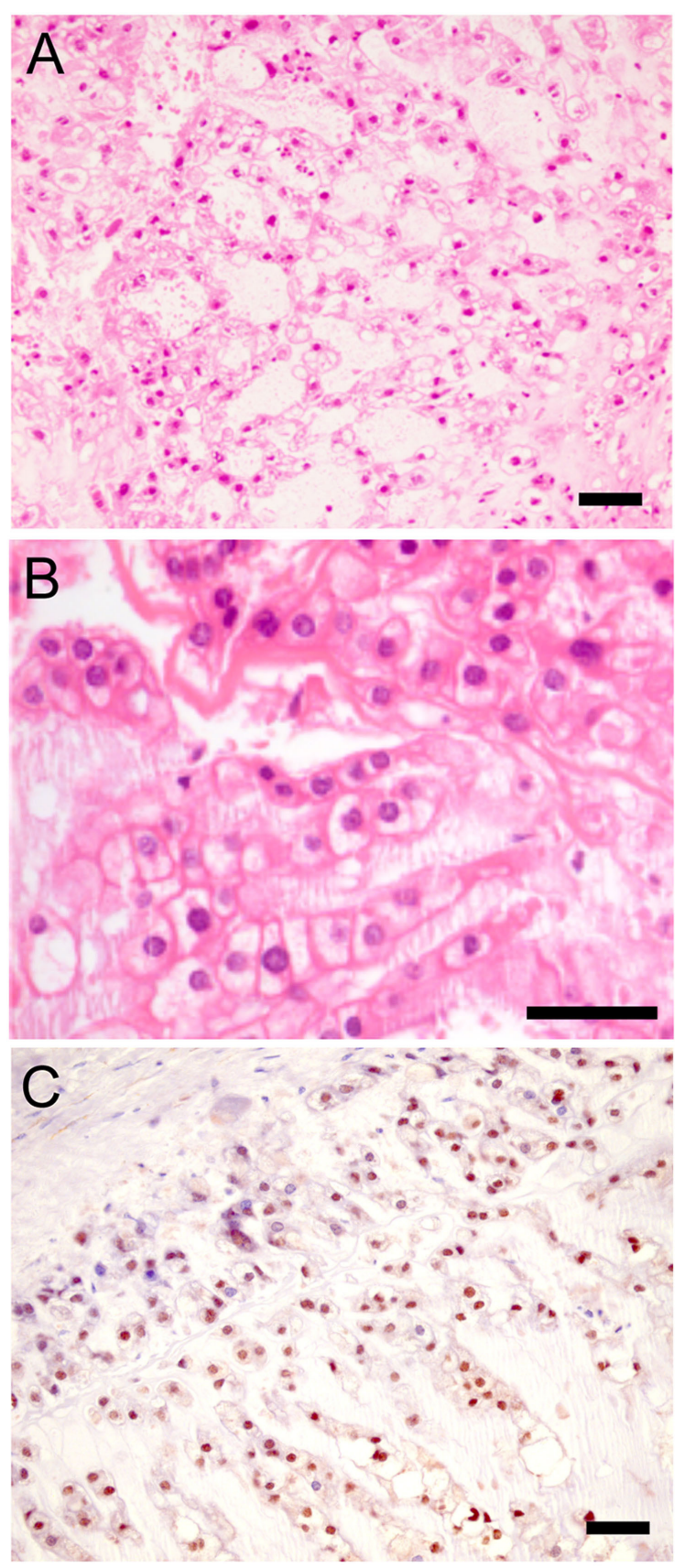

FIGURE 1 | Histological appearance of chordoma. (A,B) Sections of primary tumor stained with hematoxylin and eosin (H\&E). (C) Parallel section stained for brachyury. Scale bar: $50 \mu \mathrm{m}$.

evaluation criteria in solid tumors (RECIST) in December 2010, after 18 months of study treatment (Figures 2B,C). In February 2013, following protocol amendment to allow intra-patient dose escalation, the linsitinib dose was increased to $50 \mathrm{mg}$ BID. This treatment was monitored on a new marker lesion (left external iliac deposit, $57 \mathrm{~mm}$ ), since the previously monitored marker lesions were no longer evaluable. The patient continued on linsitinib $50 \mathrm{mg}$ BID and erlotinib $100 \mathrm{mg}$ QD with stabilization of the
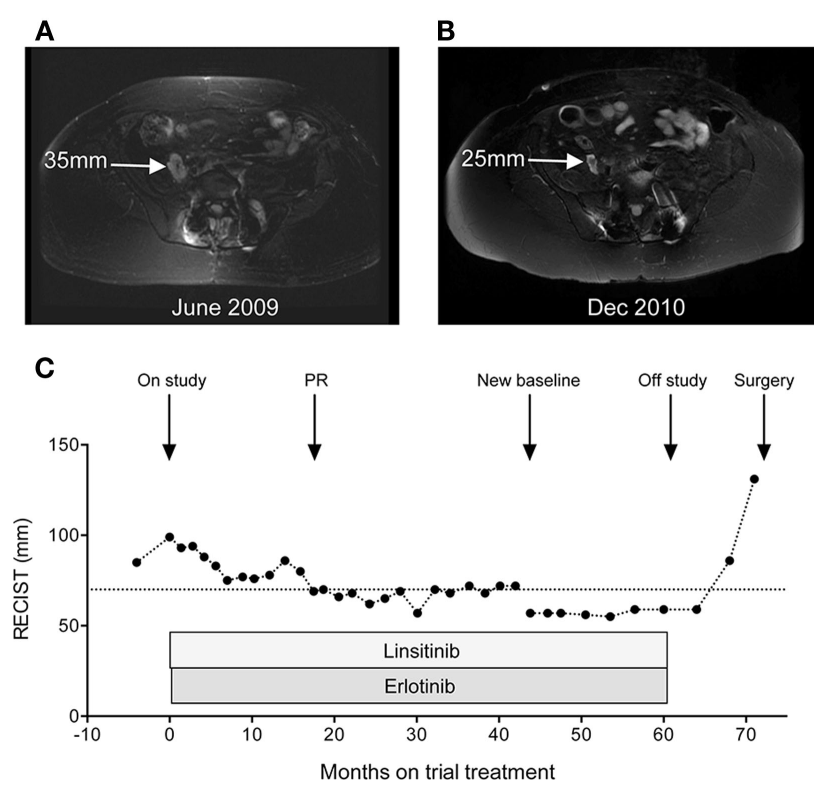

FIGURE 2 | Objective response of chordoma to linsitinib and erlotinib (A) Baseline MRI pelvis in June 2009, showing right iliopsoas deposit. Additional marker lesions were measured in the oblique right psoas $(29 \mathrm{~mm})$ and left paravertebral region $(35 \mathrm{~mm}$ ) giving a sum for the three lesions of $99 \mathrm{~mm}$. (B) Response MRI of December 2010 showing reduction in size of right iliopsoas deposit. (C) Graph: monitoring of serial MRI scans by RECIST criteria, represented as the sum of measurements of the three marker lesions described in (A). After 44 months (February 2013), these three lesions were no longer clearly measurable, and a new marker lesion (left external iliac, $57 \mathrm{~mm}$ ) was selected to monitor response to linsitinib dose escalation from $50 \mathrm{mg}$ QD to BID.

left external iliac deposit (Figure 2C). From early 2014, she experienced gradual worsening of right leg weakness. Although there was no major change in the dimensions of the monitored marker lesion, there was evidence of slow progression in non-marker lesions including deposits in the right psoas and left iliopsoas on MRI scan and left inguinal and external iliac lymphadenopathy visible on CT scan. She came off study in August 2014 after 61 months of trial treatment, and subsequently experienced further symptomatic deterioration accompanied by progression in the left external iliac deposit (Figure 2C), prompting further pelvic surgery in April 2015.

Analysis of tumor tissue was conducted under study 07/ H0606/120, approved by National Research Ethics Service (NRES) Committee South Central-Oxford C. Formalin-fixed, paraffinembedded (FFPE) tumor blocks from the 2008 surgery yielded a small amount of genomic DNA for mutation analysis. No variants were detected in EGFR codons 18, 19, and 21 (with technical failure of the exon 20 assay), KRAS, BRAF, or PIK3CA, whereas analysis of plasma DNA revealed E542K mutation in exon 9 of PIK3CA, as reported in Ref. (15). DNA was also extracted from macrodissected FFPE tumor from the 2015 resection and was tested for mutation hotspots by targeted massively parallel sequencing (http://www.oxford-translational-molecular-diagnostics.org. $\mathrm{uk} /$ ). This analysis showed no evidence of mutation in EGFR, 
TP53, PTEN, PIK3CA, PDGFRA, KRAS, NRAS, BRAF, KIT, ERBB4, FGFR1, ERBB2, MET, FGFR3, FLT3, GNAS, SMARCB1, CTNNB1, CDKN2A, ABL1, NOTCH1, PTPN11, SMO, SMAD4, VHL, NPM1, MPL, CSF1R, HRAS, JAK3, AKT1, IDH1, CDH1, FGFR2, SRC, KDR, ALK, JAK2, MLH1, RB1, HNF1A, APC, RET, STK11, FBXW7, EZH2, GNA11, GNAQ, and IDH2. A mutation of uncertain clinical significance was detected in the ATM gene [c.9031A>G, p.(M3011V), NM_000051.3].

Immunohistochemical (IHC) analysis was conducted on FFPE tumor to investigate IGF-1R and EGFR expression, using an IGF-1R IHC protocol recently optimized for sensitivity and specificity (16-18). Initial testing in FFPE control cell line pellets confirmed the specificity of the IGF-1R and EGFR antibodies in IHC (Figure 3A). Both the primary and recurrent chordoma of the trial patient contained patchy IGF-1R signal that was weaker in the primary biopsy and one of the blocks (C, psoas deposit) of recurrent tumor and stronger and more uniformly positive in two further blocks (A, B; deposits in connective tissue) from the 2008 (pre-trial) recurrence (Figure 3B). In all tumor samples, there was detectable membrane and cytoplasmic IGF-1R, with clearly apparent nuclear positivity that showed heterogeneity

\section{A}

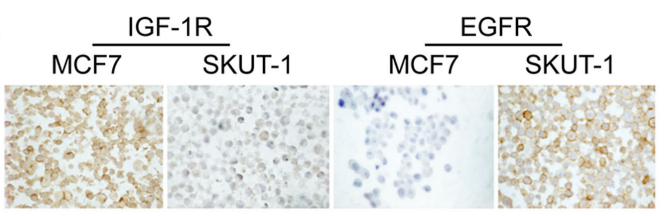

B

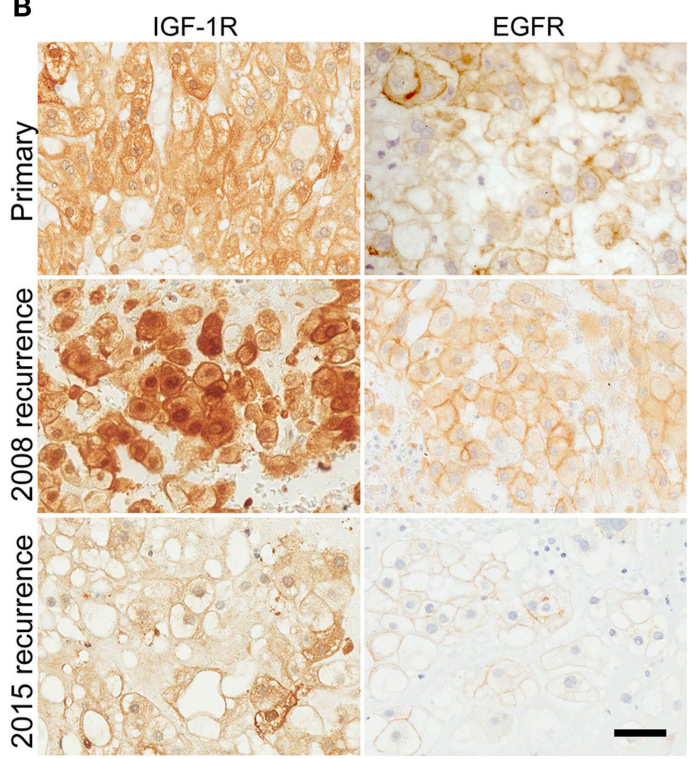

FIGURE 3 | Chordoma of trial patient contains prominent nuclear IGF-1R and membrane EGFR. (A) The specificity of IGF-1R and EGFR IHC was tested using sections of formalin-fixed, paraffin-embedded MCF7 breast cancer cells (high IGF-1R, low EGFR) and SKUT-1 leiomyosarcoma cells (IGF-1R deficient, EGFR positive). (B) Sections of chordoma from the trial patient were stained for IGF-1R and EGFR, including primary tumor from 2003, and recurrent tumor from 2008 (pre-trial) and 2015 (post-trial). Scale bar: $50 \mu \mathrm{m}$ between different regions of tumor, and was most evident in the 2008 (pre-trial) recurrence. The tumor also contained detectable EGFR, with weak/moderate membrane and cytoplasmic signal but no nuclear positivity (Figure 3B). Analysis of tumor from the 2015 (post-trial) recurrence showed that EGFR and IGF-1R were still detectable, but with reduced intensity compared with the 2008 recurrence, and reduced nuclear IGF-1R (Figure 3B).

To determine the extent to which these appearances are representative, we performed IGF-1R IHC on 15 additional chordoma cases (Figure 4), including 3 cases originating in the sacrococcygeal region and 12 cases arising in the brain. All were positive for brachyury (not shown), with heterogeneity of IGF-1R expression and subcellular localization, similar to that seen in our trial case. We scored IGF-1R in the membrane, cytoplasm, and nucleus, assessing the percentage of each sample showing zero (0), weak (1), moderate (2), or heavy (3) IGF-1R intensity. Figure 4 shows the sum of IGF-1R scores in each subcellular location, indicating that the variation in IGF-1R expression and localization in our trial case had parallels in other chordoma cases.

\section{DISCUSSION}

Previous reports have implicated receptor tyrosine kinase (RTK) signaling in the biology of chordomas (19). A recent study reported that most chordomas express multiple RTKs, including PDGFR isoforms, EGFR, HER2, c-MET, and KIT. Like IGF-1R, all of these RTKs signal via AKT to enhance tumor cell growth and survival (20), and the level of AKT phosphorylation was found to correlate with poor survival (21). In several series, which have been of limited size due to the rarity of this tumor type, imatinib-induced blockade of PDGFR and KIT has shown modest activity as monotherapy $(14,22-24)$, with evidence of activity in combination with rapamycin (25). EGFR expression has been detected by IHC in $\sim 70 \%$ of chordomas, with high level EGFR copy number gain in $40 \%$, and suppression of in vitro chordoma cell growth by a tyrphostin EGFR inhibitor (26). As in other

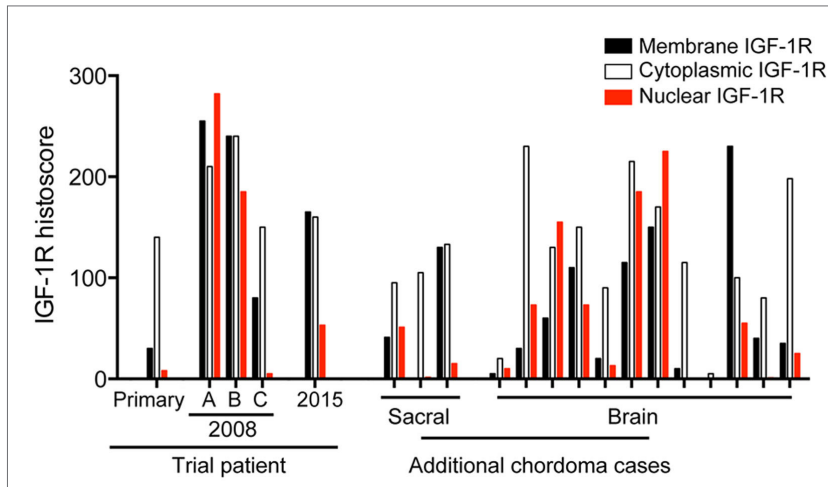

FIGURE 4 | Brain and spinal chordomas contain IGF-1R with variable membrane, cytoplasmic, and nuclear positivity. IGF-1R IHC was performed on the primary tumor, 3 tumor blocks from the 2008 recurrence and one block from the 2015 (post-trial) recurrence of the trial patient, and 15 additional chordoma cases. Each tumor was scored for IGF-1R signal in the membrane, cytoplasm, and nucleus for the percentage showing zero (0), weak (1), moderate (2), or heavy (3) IGF-1R intensity, giving a maximum histoscore in each subcellular location of $100 \% \times 3=300$. 
reported cases of chordoma, our patient's tumor lacks the EGFR mutations that are associated with response to EGFR inhibition in lung cancer $(26,27)$. A recent Phase II trial of lapatinib reported stable disease as best response (28), but there are four clinical case reports of objective responses to EGFR inhibition using erlotinib, gefitinib, and/or cetuximab (29-32). Our patient was taking $100 \mathrm{mg}$ erlotinib daily, below the recommended dose of $150 \mathrm{mg}$, although there is reported overlap in drug exposure in patients treated at 100 or $150 \mathrm{mg}$ erlotinib daily (33).

Recent studies have investigated the contribution of the IGF axis to chordoma biology. IGF-binding protein-2 (IGFBP-2) was among the genes found to be upregulated in chordoma compared with benign intervertebral disk (34). In other cellular models and tumor types IGFBP-2 has been shown to promote tumor growth and metastasis (35). Activation of IGF-1R and INSR is reportedly detectable in $\sim 40 \%$ of chordomas and is associated with significant reduction in disease-free survival (36). Another IHC study detected IGF-1R, IGF-1, and IGF-2 in 76, 90, and 50\% of chordomas, respectively, with one-third showing moderate to strong IGF-1R, as in our study, and a significant correlation between IGF-1R staining intensity and primary tumor volume (37). This study detected membrane and cytoplasmic IGF-1R but no nuclear signal (37), using an antibody to the alpha subunit of IGF-1R, which we have found is capable of detecting nuclear IGF-1R but generally gives stronger membrane signal (16). Prominent IGF-1R nuclear positivity in the tumor of our patient is of interest, given our previous finding that nuclear IGF-1R in prostate cancer models is induced by ligand and blocked by IGF-1R kinase inhibition (16). This raises the question as to whether the presence of nuclear IGF-1R indicates strong IGF axis activation, perhaps to the point where the tumor is dependent on IGF signaling. Supporting this concept, the tumors of sarcoma patients who derived benefit from therapeutic IGF-1R antibody were reported to show exclusively nuclear IGF-1R (38).

Genetic analysis of human chordomas has identified several additional changes that are implicated in tumorigenesis and could be relevant to response to therapy. First, PIK3CA (E545K) was detected in 1 of 45 patients (39) and was also detected in the circulating DNA of our patient, although its significance is

\section{REFERENCES}

1. Eriksson B, Gunterberg B, Kindblom LG. Chordoma. A clinicopathologic and prognostic study of a Swedish national series. Acta Orthop Scand (1981) 52:49-58. doi:10.3109/17453678108991758

2. Vujovic S, Henderson S, Presneau N, Odell E, Jacques TS, Tirabosco R, et al. Brachyury, a crucial regulator of notochordal development, is a novel biomarker for chordomas. J Pathol (2006) 209:157-65. doi:10.1002/ path.1969

3. Kelley MJ, Shi J, Ballew B, Hyland PL, Li WQ, Rotunno M, et al. Characterization of $\mathrm{T}$ gene sequence variants and germline duplications in familial and sporadic chordoma. Hum Genet (2014) 133:1289-97. doi:10.1007/s00439-014-1463-z

4. Yang XR, Ng D, Alcorta DA, Liebsch NJ, Sheridan E, Li S, et al. T (brachyury) gene duplication confers major susceptibility to familial chordoma. Nat Genet (2009) 41:1176-8. doi:10.1038/ng.454

5. Presneau N, Shalaby A, Ye H, Pillay N, Halai D, Idowu B, et al. Role of the transcription factor $\mathrm{T}$ (brachyury) in the pathogenesis of sporadic chordoma: a genetic and functional-based study. J Pathol (2011) 223:327-35. doi:10.1002/ path.2816 unclear, since the same mutation was not detected in the tumor. PIK3CA mutation might be predicted to mediate resistance to inhibition of signaling at the RTK level, and indeed experimental and clinical data suggest this is the case for EGFR inhibition (40, 41). The same may not be true for IGF signaling: it is known that PIK3CA-mutated breast cancer cells remain responsive to IGF stimulation and are growth delayed by IGF-1R inhibition (42-44). Second, studies of genetic alterations in chordoma identified mutation or loss of the cell cycle regulator CDKN2A $(39,45)$, which is of interest since we identified CDKN2C and CDKN3 as hits in a screen for proteins whose depletion enhances sensitivity to IGF-1R inhibition (46). Finally, our patient's tumor harbored wild-type TP53, reported by our group and others to be associated with response to small molecule IGF-1R inhibitors including linsitinib in preclinical studies $(47,48)$.

In conclusion, we report a durable objective response to linsitinib and erlotinib in a patient with spinal chordoma that had recurred following failure of local treatment. Factors that may be relevant to the response include TP53, EGFR and PIK3CA status and the presence of nuclear IGF-1R, which may signify dependence on IGF-1R and sensitivity to IGF axis inhibition. We suggest that trials incorporating assessment of these parameters should evaluate the effects of combined IGF-1R/INSR and EGFR inhibition in patients with recurrent chordomas.

\section{AUTHOR CONTRIBUTIONS}

VM, MW, ABH, SP, DW, and MM were involved in care of the patient; TA, LB, RP, SP, SH, NA, and OA contributed to data acquisition and analysis; VM drafted the manuscript, and the content was reviewed, edited, and approved by all authors.

\section{FUNDING}

MW, OA, ABH, MM, and VM are supported by the NIHR Oxford Biomedical Research Centre, and $\mathrm{ABH}$ by the NIHR Oxford Musculoskeletal BRU. Work in the laboratory of VM is supported by Breast Cancer Now, Prostate Cancer UK, Rosetrees Trust, UCARE-Oxford, and Oxford Cancer Research Centre.

6. Pillay N, Plagnol V, Tarpey PS, Lobo SB, Presneau N, Szuhai K, et al. A common single-nucleotide variant in $\mathrm{T}$ is strongly associated with chordoma. Nat Genet (2012) 44:1185-7. doi:10.1038/ng.2419

7. Bergh P, Kindblom LG, Gunterberg B, Remotti F, Ryd W, MeisKindblom JM. Prognostic factors in chordoma of the sacrum and mobile spine: a study of 39 patients. Cancer (2000) 88:2122-34. doi:10.1002/ (SICI)1097-0142(20000501)88:9<2122::AID-CNCR19>3.0.CO;2-1

8. McMaster ML, Goldstein AM, Bromley CM, Ishibe N, Parry DM. Chordoma: incidence and survival patterns in the United States, 1973-1995. Cancer Causes Control (2001) 12:1-11. doi:10.1023/A:1008947301735

9. Chugh R, Tawbi H, Lucas DR, Biermann JS, Schuetze SM, Baker LH. Chordoma: the nonsarcoma primary bone tumor. Oncologist (2007) 12:1344-50. doi:10.1634/theoncologist.12-11-1344

10. Hanna SA, Aston WJ, Briggs TW, Cannon SR, Saifuddin A. Sacral chordoma: can local recurrence after sacrectomy be predicted? Clin Orthop Relat Res (2008) 466:2217-23. doi:10.1007/s11999-008-0356-7

11. Garofalo F, Christoforidis D, di Summa PG, Gay B, Cherix S, Raffoul W, et al. The unresolved case of sacral chordoma: from misdiagnosis to challenging surgery and medical therapy resistance. Ann Coloproctol (2014) 30:122-31. doi:10.3393/ac.2014.30.3.122 
12. Tauziède-Espariat A, Bresson D, Polivka M, Bouazza S, Labrousse F, Aronica E, et al. Prognostic and therapeutic markers in chordomas: a study of 287 tumors. J Neuropathol Exp Neurol (2016). doi:10.1093/jnen/nlv010

13. Bydon M, Papadimitriou K, Witham T, Wolinsky JP, Bydon A, Sciubba D, et al. Novel therapeutic targets in chordoma. Expert Opin Ther Targets (2012) 16:1139-43. doi:10.1517/14728222.2012.714772

14. Walcott BP, Nahed BV, Mohyeldin A, Coumans JV, Kahle KT, Ferreira MJ. Chordoma: current concepts, management, and future directions. Lancet Oncol (2012) 13:e69-76. doi:10.1016/S1470-2045(11)70337-0

15. Macaulay VM, Middleton MR, Eckhardt SG, Rudin CM, Juergens RA, Gedrich R, et al. Phase I dose-escalation study of linsitinib (OSI-906) and erlotinib in patients with advanced solid tumors. Clin Cancer Res (2016). doi:10.1158/1078-0432.CCR-15-2218

16. Aleksic T, Chitnis MM, Perestenko OV, Gao S, Thomas PH, Turner GD, et al. Type 1 insulin-like growth factor receptor translocates to the nucleus of human tumor cells. Cancer Res (2010) 70:6412-9. doi:10.1158/0008-5472. CAN-10-0052

17. Dale OT, Aleksic T, Shah KA, Han C, Mehanna H, Rapozo DC, et al. IGF-1R expression is associated with HPV-negative status and adverse survival in head and neck squamous cell cancer. Carcinogenesis (2015) 36:648-55. doi:10.1093/ carcin/bgv053

18. Aleksic T, Worrall AR, Verrill C, Turley H, Campo L, Macaulay VM. Improved immunohistochemical detection of type 1 insulin-like growth factor receptor in human tumors. Immunochem Immunopathol (2016) 2:114. doi:10.4172/2469-9756.1000114

19. Tamborini E, Virdis E, Negri T, Orsenigo M, Brich S, Conca E, et al. Analysis of receptor tyrosine kinases (RTKs) and downstream pathways in chordomas. Neuro Oncol (2010) 12:776-89. doi:10.1093/neuonc/noq003

20. Chitnis MM, Yuen JS, Protheroe AS, Pollak M, Macaulay VM. The type 1 insulin-like growth factor receptor pathway. Clin Cancer Res (2008) 14:6364-70. doi:10.1158/1078-0432.CCR-07-4879

21. de Castro CV, Guimaraes G, Aguiar S Jr, Lopes A, Baiocchi G, da Cunha IW, et al. Tyrosine kinase receptor expression in chordomas: phosphorylated AKT correlates inversely with outcome. Hum Pathol (2013) 44:1747-55. doi:10.1016/j.humpath.2012.11.024

22. Casali PG, Messina A, Stacchiotti S, Tamborini E, Crippa F, Gronchi A, et al. Imatinib mesylate in chordoma. Cancer (2004) 101:2086-97. doi:10.1002/ cncr.20618

23. Ferraresi V, Nuzzo C, Zoccali C, Marandino F, Vidiri A, Salducca N, et al. Chordoma: clinical characteristics, management and prognosis of a case series of 25 patients. BMC Cancer (2010) 10:22. doi:10.1186/1471-2407-10-22

24. Stacchiotti S, Longhi A, Ferraresi V, Grignani G, Comandone A, Stupp R, et al. Phase II study of imatinib in advanced chordoma. J Clin Oncol (2012) 30:914-20. doi:10.1200/JCO.2011.35.3656

25. Stacchiotti S, Marrari A, Tamborini E, Palassini E, Virdis E, Messina A, et al. Response to imatinib plus sirolimus in advanced chordoma. Ann Oncol (2009) 20:1886-94. doi:10.1093/annonc/mdp210

26. Shalaby A, Presneau N, Ye H, Halai D, Berisha F, Idowu B, et al. The role of epidermal growth factor receptor in chordoma pathogenesis: a potential therapeutic target. J Pathol (2011) 223:336-46. doi:10.1002/path.2818

27. Pao W, Chmielecki J. Rational, biologically based treatment of EGFR-mutant non-small-cell lung cancer. Nat Rev Cancer (2010) 10:760-74. doi:10.1038/ nrc2947

28. Stacchiotti S, Tamborini E, Lo Vullo S, Bozzi F, Messina A, Morosi C, et al. Phase II study on lapatinib in advanced EGFR-positive chordoma. Ann Oncol (2013) 24:1931-6. doi:10.1093/annonc/mdt117

29. Hof H, Welzel T, Debus J. Effectiveness of cetuximab/gefitinib in the therapy of a sacral chordoma. Onkologie (2006) 29:572-4. doi:10.1159/000096283

30. Linden O, Stenberg L, Kjellen E. Regression of cervical spinal cord compression in a patient with chordoma following treatment with cetuximab and gefitinib. Acta Oncol (2009) 48:158-9. doi:10.1080/02841860802266672

31. Singhal N, Kotasek D, Parnis FX. Response to erlotinib in a patient with treatment refractory chordoma. Anticancer Drugs (2009) 20:953-5. doi:10.1097/ CAD.0b013e328330c7f0

32. Launay SG, Chetaille B, Medina F, Perrot D, Nazarian S, Guiramand J, et al. Efficacy of epidermal growth factor receptor targeting in advanced chordoma: case report and literature review. BMC Cancer (2011) 11:423. doi:10.1186/1471-2407-11-423

33. Hidalgo M, Siu LL, Nemunaitis J, Rizzo J, Hammond LA, Takimoto C, et al. Phase I and pharmacologic study of OSI-774, an epidermal growth factor receptor tyrosine kinase inhibitor, in patients with advanced solid malignancies. J Clin Oncol (2001) 19:3267-79.

34. Scheil-Bertram S, Kappler R, von Baer A, Hartwig E, Sarkar M, Serra M, et al. Molecular profiling of chordoma. Int J Oncol (2014) 44:1041-55. doi:10.3892/ ijo. 2014.2268

35. Baxter RC. IGF binding proteins in cancer: mechanistic and clinical insights. Nat Rev Cancer (2014) 14:329-41. doi:10.1038/nrc3720

36. Sommer J, Itani DM, Homlar KC, Keedy VL, Halpern JL, Holt GE, et al. Methylthioadenosine phosphorylase and activated insulin-like growth factor-1 receptor/insulin receptor: potential therapeutic targets in chordoma. J Pathol (2010) 220:608-17. doi:10.1002/path.2679

37. Scheipl S, Froehlich EV, Leithner A, Beham A, Quehenberger F, Mokry $\mathrm{M}$, et al. Does insulin-like growth factor 1 receptor (IGF-1R) targeting provide new treatment options for chordomas? A retrospective clinical and immunohistochemical study. Histopathology (2012) 60:999-1003. doi:10.1111/j.1365-2559.2012.04186.x

38. Asmane I, Watkin E, Alberti L, Duc A, Marec-Berard P, Ray-Coquard I, et al. Insulin-like growth factor type 1 receptor (IGF-1R) exclusive nuclear staining: a predictive biomarker for IGF-1R monoclonal antibody (Ab) therapy in sarcomas. Eur J Cancer (2012) 48:3027-35. doi:10.1016/j.ejca.2012.05.009

39. Choy E, MacConaill LE, Cote GM, Le LP, Shen JK, Nielsen GP, et al. Genotyping cancer-associated genes in chordoma identifies mutations in oncogenes and areas of chromosomal loss involving CDKN2A, PTEN, and SMARCB1. PLoS One (2014) 9:e101283. doi:10.1371/journal.pone.0101283

40. Engelman JA, Mukohara T, Zejnullahu K, Lifshits E, Borras AM, Gale CM, et al. Allelic dilution obscures detection of a biologically significant resistance mutation in EGFR-amplified lung cancer. J Clin Invest (2006) 116:2695-706. doi:10.1172/JCI28656

41. Ohashi K, Maruvka YE, Michor F, Pao W. Epidermal growth factor receptor tyrosine kinase inhibitor-resistant disease. J Clin Oncol (2013) 31:1070-80. doi:10.1200/JCO.2012.43.3912

42. Frogne T, Jepsen JS, Larsen SS, Fog CK, Brockdorff BL, Lykkesfeldt AE. Antiestrogen-resistant human breast cancer cells require activated protein kinase B/Akt for growth. Endocr Relat Cancer (2005) 12:599-614. doi:10.1677/ erc. 1.00946

43. Lisztwan J, Pornon A, Chen B, Chen S, Evans DB. The aromatase inhibitor letrozole and inhibitors of insulin-like growth factor I receptor synergistically induce apoptosis in in vitro models of estrogen-dependent breast cancer. Breast Cancer Res (2008) 10:R56. doi:10.1186/bcr2113

44. Baxi SM, Tan W, Murphy ST, Smeal T, Yin MJ. Targeting 3-phosphoinoside-dependent kinase-1 to inhibit insulin-like growth factor-I induced AKT and p70 S6 kinase activation in breast cancer cells. PLoS One (2012) 7:e48402. doi:10.1371/journal.pone.0048402

45. Le LP, Nielsen GP, Rosenberg AE, Thomas D, Batten JM, Deshpande V, et al. Recurrent chromosomal copy number alterations in sporadic chordomas. PLoS One (2011) 6:e18846. doi:10.1371/journal.pone.0018846

46. Gao S, Bajrami I, Verrill C, Kigozi A, Ouaret D, Aleksic T, et al. Dsh homolog DVL3 mediates resistance to IGFIR inhibition by regulating IGF-RAS signaling. Cancer Res (2014) 74:5866-77. doi:10.1158/0008-5472.CAN-14-0806

47. Wang Q, Wei F, Lv G, Li C, Liu T, Hadjipanayis CG, et al. The association of TP53 mutations with the resistance of colorectal carcinoma to the insulin-like growth factor-1 receptor inhibitor picropodophyllin. BMC Cancer (2013) 13:521. doi:10.1186/1471-2407-13-521

48. Ramcharan R, Aleksic T, Kamdoum WP, Gao S, Pfister SX, Tanner J, et al. IGF-1R inhibition induces schedule-dependent sensitization of human melanoma to temozolomide. Oncotarget (2015) 6:39877-90. doi:10.18632/ oncotarget.5631

Conflict of Interest Statement: The authors declare that the research was conducted in the absence of any commercial or financial relationships that could be construed as a potential conflict of interest.

Copyright (C) 2016 Aleksic, Browning, Woodward, Phillips, Page, Henderson, Athanasou, Ansorge, Whitwell, Pratap, Hassan, Middleton and Macaulay. This is an open-access article distributed under the terms of the Creative Commons Attribution License (CC BY). The use, distribution or reproduction in other forums is permitted, provided the original author(s) or licensor are credited and that the original publication in this journal is cited, in accordance with accepted academic practice. No use, distribution or reproduction is permitted which does not comply with these terms. 